\title{
www.smf-cme.ch: Feedback verbessert die medizinische Fortbildung
}

\author{
M. Otto, N. Marty, S. Walser
}

Leserinnen und Leser des Swiss Medical Forum (SMF) können ihr Wissen anhand von Multiple-choice-Fragen, die sich auf Fortbildungsbeiträge im SMF beziehen, überprüfen. Die Fragen stehen im Heft und gleichzeitig online zur Verfügung. Die Beantwortung der Fragen online erlaubt, sofort die richtige Antwort zu erfahren und einen kurzen ergänzenden Kommentar zu lesen. Damit kann der persönliche Lernerfolg geprüft werden. Zudem kann die Redaktion durch Auswertung der Fragen feststellen, ob einzelne Fragen statistisch auffällig sind, und diese bei Bedarf überarbeiten.

\section{Abbildung 1}

www.smf-cme.ch.

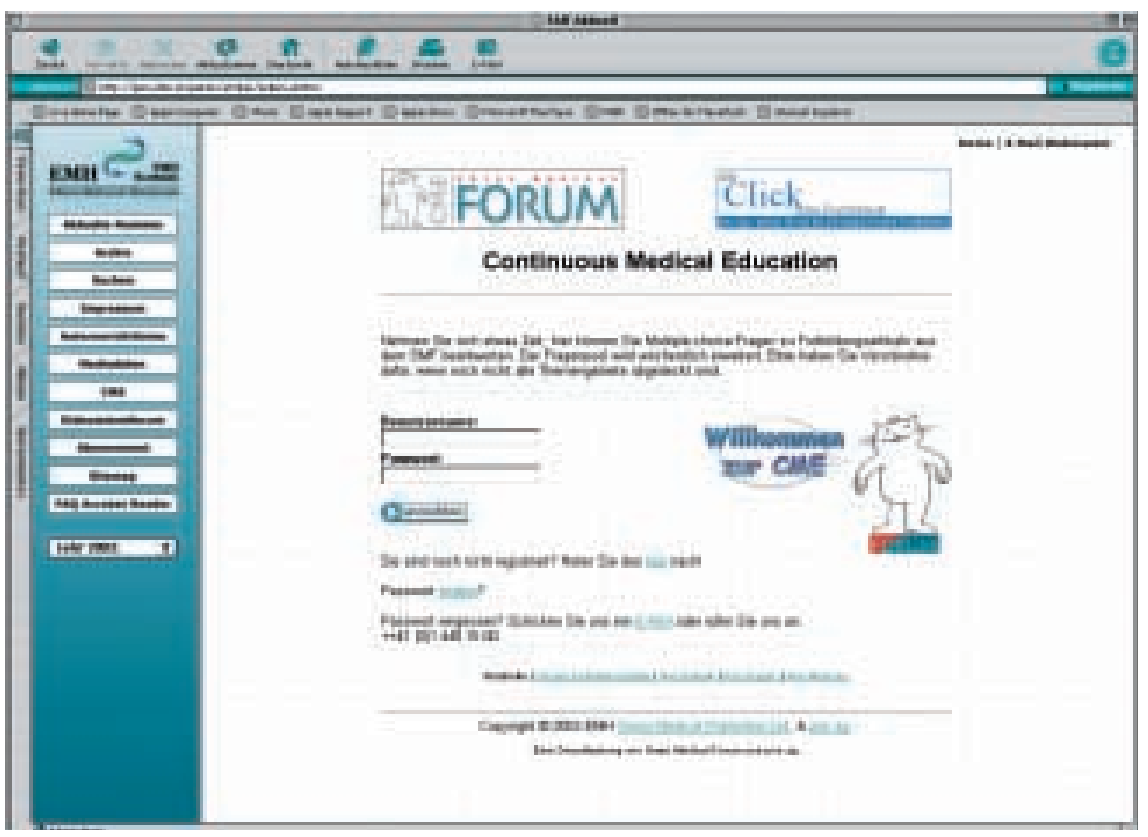

Korrespondenz:

Dr. oec. publ. Marc Otto

pnn ag

Technopark Zürich

Technoparkstrasse 1

CH-8005 Zürich

Tel. 014451500

Dr. med. Natalie Marty,

Dr. med. Susanna Walser-Uebersax

EMH Schweizerischer Ärzteverlag AG

Steinentorstrasse 13

CH-4010 Basel

\section{Websites:}

www.emh.ch, www.medicalforum.ch www.smf-cme.ch, http://pnn.ethz.ch kommen 30 Stunden Selbststudium, was zusammengenommen 10 Tagen Fortbildung pro Jahr entspricht.

Das Lesen von Fachzeitschriften und auch das Beantworten artikelbezogener Multiple-choiceFragen wird als Selbststudium bewertet, solange kein Nachweis dafür erbracht wird. Allerdings besteht die Möglichkeit, so geleistete Fortbildung festzuhalten und anzurechnen; Voraussetzungen dazu sind einerseits das Registrieren der Leser und das Erfassen der Antworten sowie andererseits die Akkreditierung und Zertifizierung durch eine medizinische Fachgesellschaft. Solche CME-Angebote werden nun weltweit von den medizinischen Zeitschriften lanciert. In Deutschland z. B. bieten verschiedene Fachzeitschriften seit einiger Zeit zertifizierte Fortbildung an. $\mathrm{Zu}$ jedem Fortbildungsartikel werden vertiefende Multiple-choice-Fragen zusammen mit einem Antwortformular abgedruckt. Genügte es bis vor kurzem, das Antwortformular ausgefüllt einzureichen, so müssen seit Anfang dieses Jahres $70 \%$ der Fragen richtig beantwortet sein, damit Credits erworben werden können. Immer mehr Zeitschriften gehen mittlerweile dazu über, dass die Leser ihre Antworten nicht faxen oder einschicken, sondern diese in ein Online-Formular eintragen.

\section{Technische Anforderungen für Print- und Online-Fortbildungen}

Die Teilnahme an einer Online-Fortbildung muss festgehalten, also registriert, werden, um ein Fortbildungszertifikat und Kreditpunkte dafür ausstellen zu können. Die Zertifikate sind meistens an Bedingungen der Fachgesellschaften gebunden, deren Einhaltung durch den Anbieter sichergestellt sein muss.

In seiner einfachsten Form ist ein Antwortformular stufenweise aufgebaut, so dass der Benutzer erst dann eine neue Frage einsehen kann, wenn er die vorhergehende richtig beantwortet hat. So arbeitet sich der Benutzer schrittweise durch die Fortbildung, bis er zum Link, mit welchem das Zertifikat abgerufen werden kann, gelangt. 
Abbildung 2

Gliederung der Artikel nach Themengebieten.

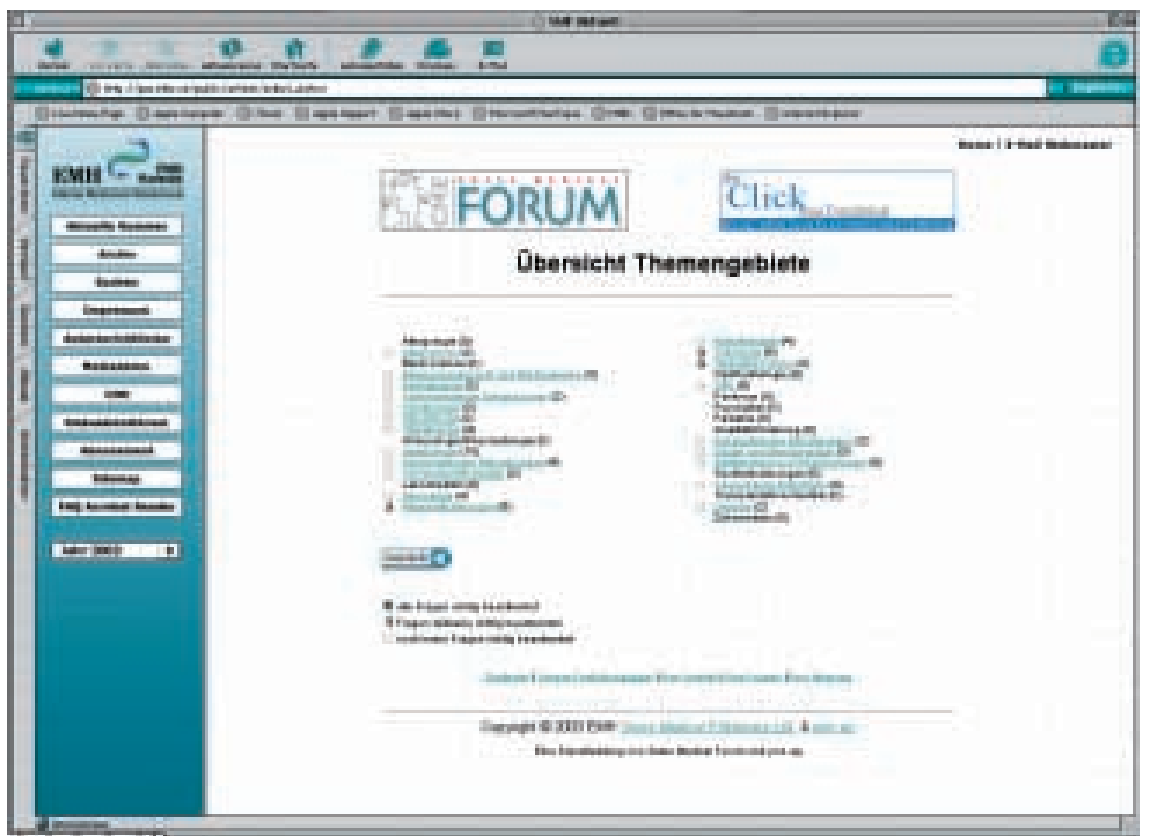

Abbildung 3

Die Abbildung links zeigt, wie die Teilnehmer mit dem besten Resultat bei allen Fragen in einem gesamten Fachgebiet abgeschlossen haben. Die Abbildung rechts zeigt, wie dieselbe Gruppe auf eine bestimmte Frage geantwortet hat. Da das erzielte Resultat der Gruppe so schlecht ist (viel falsch), könnte die Frage schlecht formuliert sein.
Top 10\% bei allen Fragen

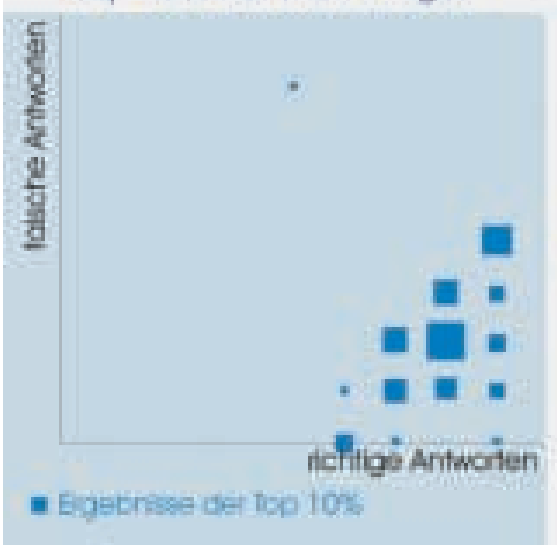

\section{Resultate bei einzelner Frage}

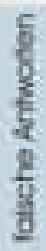

fontige Antwoten
Print- und Online-Fortbildung im Swiss Medical Forum

Die Artikel in der Rubrik «Curriculum» des Swiss Medical Forum decken über einen Zeitraum von 3 Jahren die wichtigsten Themen der grossen internistischen Fachgebiete ab. Die Rubrik «Praxis» behandelt wichtige Themen der hausärztlichen Diagnostik und Therapie; auch hier arbeitet die Redaktion nach einem 3-Jahres-Plan. Jeder Fortbildungsartikel wird im Verlag nicht nur nach den üblichen Suchkriterien wie Titel, Autor oder Keywords indexiert, sondern auch nach den Themengebieten.

Die Print- und Online-Fortbildung des Swiss Medical Forum - www.smf-cme.ch (Abb. 1) wurde gemeinsam durch den Schweizerischen Ärzteverlag EMH und pnn ag, einem Spin-offUnternehmen der ETH Zürich, entwickelt. Sie basiert auf einer leistungsstarken Datenbank.

Für die Leserinnen und Leser bietet diese Website einen Kanal für Feedback und eine Übersicht des eigenen Lernfortschritts mitsamt Benchmarking. Zur Online-Fortbildung kann man nach Identifikation mit Benutzername und Passwort über die Liste der bereits erschienenen Hefte einsteigen. Mit einem Klick erscheinen die Fragen zu den entsprechenden Artikeln, welche schrittweise durchgearbeitet werden können. Jede Antwort wird sofort ausgewertet und mit einer kurzen Erklärung kommentiert. Sollte der Kommentar nicht ausreichen, kann mit einem Klick der entsprechende Fortbildungsartikel abgerufen werden, da alle Artikel im Volltext online zur Verfügung stehen. Sind sämtliche Fragen beantwortet, erscheint eine Auswertung, eine Benchmark, welche das eigene mit dem gemittelten Ergebnis aller anderen Teilnehmer vergleicht.

Die Gliederung der Artikel nach Themengebieten (Abb. 2) erlaubt aber auch einen anderen Zugang zu den Fortbildungsartikeln: Die Fragen werden hier nach medizinischen Themengebieten geordnet aufgelistet. So kann man jeweils wählen, zu welchem Fachgebiet man in einer Online-Sitzung Fragen abarbeiten will. War eine Antwort falsch oder unklar, kann man natürlich auch hier den zur Frage gehörenden Artikel nachlesen, um die Wissenslücke zu schliessen. Am Ende der Fragen zu einem Themengebiet erscheint wiederum eine Benchmark.

Als Hinweis, wie gut man in den verschiedenen Gebieten abgeschnitten hat, wird angezeigt, ob man die entsprechenden Fragen bereits alle richtig, teilweise richtig oder noch gar nicht beantwortet hat. 


\section{Aufzeichnungen zwecks Qualitäts- sicherung und Datenschutz}

Das Lernverhalten aller Benutzer/innen wird von der Lernplattform anonymisiert und statistisch ausgewertet. Dies ist wichtig, um mit einem Quervergleich (Abb. 3) auffällige Fragen herauszufinden und anschliessend bei Bedarf zu überarbeiten. In einem ersten Schritt wird dafür eine Benutzergruppe zusammengestellt, welche insgesamt am meisten Fragen richtig beantwortet hat. Dann wird analysiert, wie diese Gruppe bei jeder einzelnen Frage abgeschnitten hat. Erscheint dabei eine auffällige Diskrepanz, wird die entsprechende Frage nochmals überprüft.

Gehäuft falsch beantwortete Fragen zeigen auf, dass diese den Leserinnen und Lesern besondere Mühe bereiten, weil sie beispielsweise zu schwierig oder zu wenig klar formuliert sind oder weil die Antworten inhaltlich nicht eindeutig als richtig oder falsch zu erkennen sind.

Fragen, die von den meisten Benutzer/innen richtig beantwortet werden, sind möglicherweise zu einfach oder durch Überfliegen des Artikels oder der Quintessenz bereits zu beantworten.

Gute Multiple-choice-Fragen sollten zudem zwischen erfolgreichen Benutzern und solchen mit Wissenslücken diskriminieren können.

Um Wissenslücken aufzudecken, werden die Antworten der verschiedenen Themengebiete miteinander verglichen. Damit kann die Redaktion Hinweise gewinnen, für welche Gebiete möglicherweise ein grösserer Fortbildungsbedarf besteht. So kann die Online-Plattform, welche ja massgeblich von den publizierten Artikeln abhängt, der Fachzeitschrift wiederum wertvolle Rückmeldungen geben.

Basis dieser Auswertungen sind personenbezogene Daten, welche nach den geltenden Richtlinien des Datenschutzes behandelt werden. Um sie auch technisch wirksam zu schützen, werden exponiertere Benutzerdaten, wie Passwörter, nur verschlüsselt auf dem Server abgelegt. Können personenbezogene Daten nicht anonymisiert werden, z.B. bei der erstmaligen Registrierung, so wird die Übertragung mit 128-Bit-Verschlüsselung geschützt, dem Sicherheitsstandard, welcher auch beim Online-Banking verwendet wird.

\section{Ausblick}

Ein Wunsch der Redaktion des Swiss Medical Forum ist es, dass die Fortbildungsfragen nicht nur als eine «Pflichtübung» betrachtet werden, sondern auch unterhalten und «Aha»-Erlebnisse hervorrufen. In naher Zukunft soll aber die Akkreditierung der Online-Fortbildung durch medizinische Fachgesellschaften den Mitgliedern dieser FG auch die Möglichkeit geben, durch Lektüre der Zeitschrift und Beantworten der CME-Fragen auch direkt Credits zu sammeln und entsprechende Fortbildungszertifikate zu erhalten. Darüber wird in einigen Wochen im Swiss Medical Forum selber mehr zu lesen sein. 